\title{
Interhemispheric Approach
}

\author{
Alberto Feletti, Dilshod Mamadaliev, \\ Tushit Mewada, Kei Yamashiro, Yasuhiro Yamada, \\ Tsukasa Kawase, and Yoko Kato
}

\subsection{Introduction}

The interhemispheric (IH) approach is the best access to lesions located in the midline along the corpus callosum and can be used also for lesions

\footnotetext{
A. Feletti $(\bowtie)$

Department of Neurosurgery, Fujita Health University, Banbuntane Hotokukai Hospital, Nagoya city, Aichi, Japan
}

Department of Neurosurgery, NOCSAE Modena Hospital, Modena, Italy

D. Mamadaliev

Department of Neurosurgery, Fujita Health University, Banbuntane Hotokukai Hospital, Nagoya city, Aichi, Japan

Republican Scientific Center of Neurosurgery, Tashkent, Uzbekistan

\section{T. Mewada}

Department of Neurosurgery, Fujita Health University, Banbuntane Hotokukai Hospital, Nagoya city, Aichi, Japan

G. B. Pant Institute of Post Graduate Medical Education and Research, New Delhi, India

K. Yamashiro · Y. Yamada · T. Kawase Department of Neurosurgery, Fujita Health University, Banbuntane Hotokukai Hospital, Nagoya city, Aichi, Japan e-mail: kawasemi@hm8.aitai.ne.jp

\section{Y. Kato}

Department of Neurosurgery, Fujita Health University, Toyoake, Aichi, Japan e-mail: kyoko@fujita-hu.ac.jp in the lateral and third ventricles and in the pineal region.

Depending on the position of the target along the anteroposterior direction, the skin incision and craniotomy can vary. However, as the majority of distal anterior cerebral artery (DACA) aneurysms located along the corpus callosum especially the rostrum part, the anterior approach represents the most common way to reach them. The approach may minimally displace the frontal lobe to get enough space to expose the falx, the anterior cerebral arteries, and the corpus callosum.

Unilateral low anterior IH approach is a variation, which allows reaching the anterior communicating artery $(\mathrm{ACoA})$ aneurysms.

\subsection{Steps of the Approach}

\subsubsection{Positioning}

As for other approaches, patient position is supine with the head and shoulders slightly higher than the heart to reduce bleeding by facilitating cerebral venous drainage. The head is fixed with either Mayfield (three pins) or Sugita (four pins) head holder, in straight position, and either with slight extension or flexion to get the best projection to the lesion. The degree of head flexion and extension depends on the relative position of the pathology compared to rostral part of 
corpus callosum. More distal lesions require more flexion of the head, whereas lesions located close to the rostral part may just be approached with head in neutral position.

In the unilateral anterior IH approach, the patient's head is rotated to orient the midline horizontally and tilted $45^{\circ}$ superiorly.

\subsubsection{Incision}

After minimal or no shaving, a frontal scalp incision is made starting just behind the hairline, $1 \mathrm{~cm}$ beyond the midline. The incision extends backwards parallel to the midline for $4-5 \mathrm{~cm}$, bends laterally to cross the midline, and prolongs for $4-5 \mathrm{~cm}$. The skin-galea flap is elevated and retracted anterolaterally with skin hooks. At the edge of skin incision, we may apply Raney clips for hemostasis.

Alternatively, a bicoronal skin incision can be performed just behind the hairline from one side to the other, starting $2 \mathrm{~cm}$ above the zygomatic arc. The temporalis muscle must be preserved.

\subsubsection{Craniotomy}

Start with two burr holes at the anterior and posterior margin of the bone flap, preferable exactly to expose the midline. If necessary, additional lateral burr holes can be placed if the dura adheres very tight to the inner table of the bone (Fig. 5.1). The dura is released from the inner table of the bone, using curved and blunt dissector. Craniotomy is then performed using a sidecutting craniotome with a footplate to obtain an approximately $4 \mathrm{~cm} \times 4 \mathrm{~cm}$ bone flap encompassing the median line. In case of unilateral craniotomy, it is advisable to leave the median side of the craniotomy as the last step, as it has the highest risk for dural damage and consequent superior sagittal sinus opening. Actually, if that happens, less time would be required to elevate the bone flap and compress the sagittal sinus to reduce blood loss.

In the unilateral anterior (basal) IH approach, the bone flap is placed more frontally, as the skin is retracted anteriorly to a wider extent in order to obtain a more favorable direction toward the ACoA under the rostrum of the corpus callosum. Efforts should be made to preserve the frontal sinus. The bone flap is then elevated using a blunt dissector (Fig. 5.2).

Before opening the dura, the margins of the skin incision are covered with povidone-iodinesoaked towels, which can be secured to the skin using Raney clips.

Surgeon open the dura with a "U" shape, and the base of the flap lying along the superior sagittal sinus (Fig. 5.3). The two branches of the dural incision extend to the limit of the sagittal sinus, avoiding its violation. If the sagittal sinus is inadvertently opened at this stage, a dural clip can be placed to stop the bleeding. Two or three tack-up sutures are placed to retract the dural edge toward

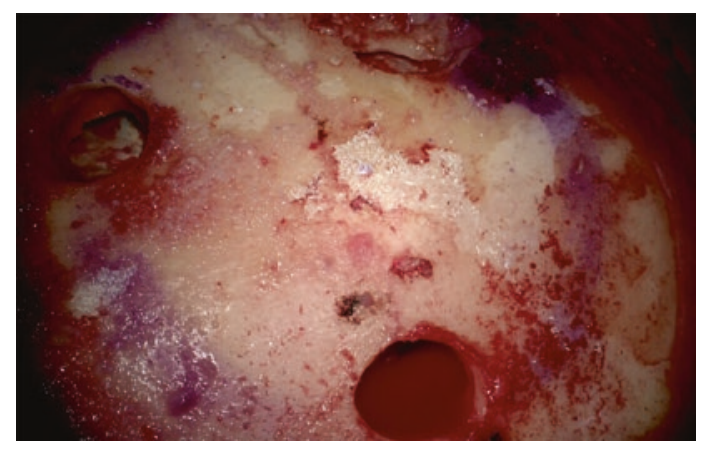

Fig. 5.1 Two burr holes are placed at the most anterior and posterior portion of the exposed midline. Occasionally, a third lateral burr hole can be placed when the dura is not easily detachable from the inner theca of the skull

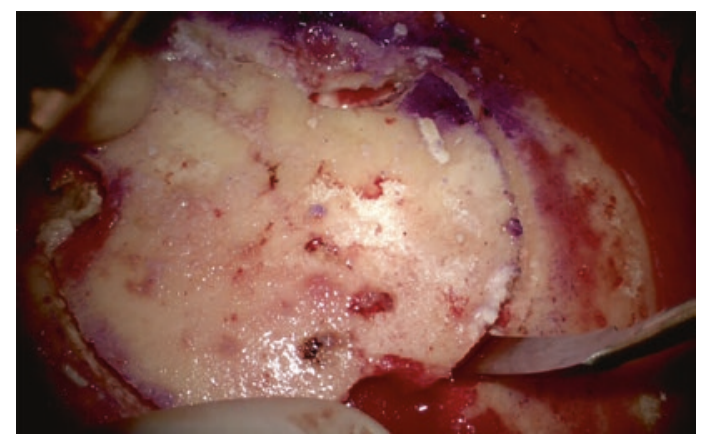

Fig. 5.2 The bone flap is elevated using a blunt dissector 


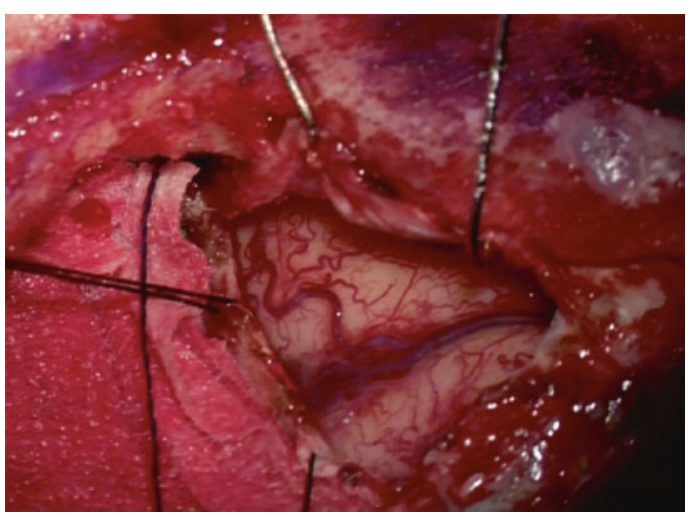

Fig. 5.3 The dura is opened and elevated toward the median line (on the left)

the median craniotomy margin. When approaching an ACoA aneurysm, sometimes a wider exposure is needed depending on the anatomical characteristics of aneurysm and vessels. In this case, the superior sagittal sinus can be closed using stiches placed at its most anterior part in the proximity of crista galli, and then cut. The dura and the falx can be therefore retracted posteriorly. The surgical microscope is placed so the surgeon may have all the flexibility for surgical maneuver.

Before beginning dissection of interhemispheric fissure, it is necessary to identify the best entry point and direction. Usually, the entry point is chosen in the space between the eventual bridging veins, to minimize vascular traction and damage. Neuronavigation systems provide a valuable tool to detect the appropriate direction of dissection. As the brain is progressively detached from the falx, a retractor can be placed to mildly retract the frontal lobe obtaining wider space and better vision along the falx. In the unilateral anterior $\mathrm{IH}$ approach, there is no need for active retraction as gravity itself pushes the frontal lobe downward. In any case, the cerebrospinal fluid (CSF) release obtained with subarachnoid dissection allows gradual brain relaxation and wider surgical exposure.

The dissection proceeds along the falx until either the ACAs or the ACoA are visualized (Fig. 5.4). In case of ACoA aneurysms, crista galli must be removed to obtain a wide operative field and full control of vessels.

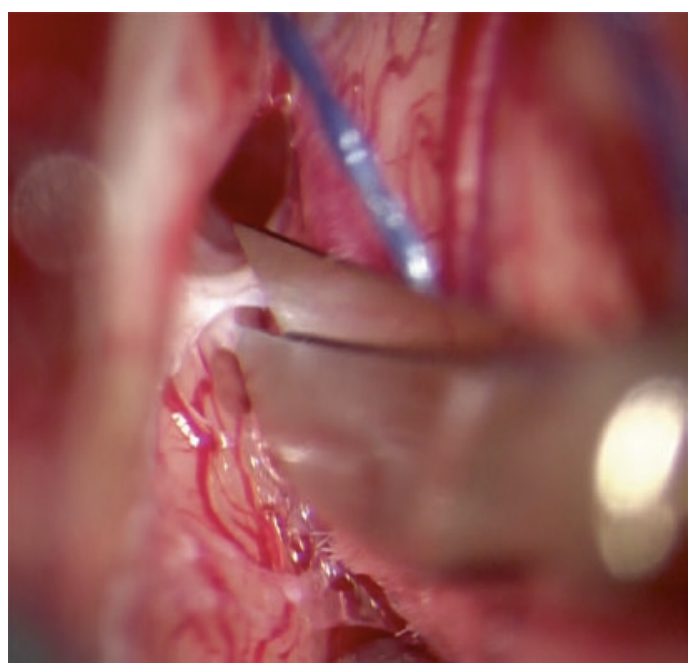

Fig. 5.4 Following the direction of the falx (on the left), subarachnoid dissection is performed

\subsubsection{Closure}

Before closure, any retractor must be removed and the underlying brain must be visualized in order to detect and block eventual bleedings. The dura is tightly closed in a waterproof manner with a resorbable filament (Safil 3-0 or 4-0), eventually adding fibrin glue along the dural suture. Suture should begin from the lower end of the dural opening and proceed to the upper end. In this way, just before the very last sutures, a blunt needle can be inserted intradurally to fill the subdural space with saline and remove the air. Additional tack-up sutures can be placed at the dura to the craniotomy edge, to minimize the risk of postoperative epidural hematoma. It is advisable to avoid placing these sutures along the median side of the craniotomy to prevent any puncture of the superior sagittal sinus.

The bone flap is replaced and fixed using either microplates and microscrews or sutures placed between the previously drilled holes around the craniotomy edges and along the bone flap margins. The galea and subcutaneous layer are sutured simultaneously using separate sutures with resorbable filament (Vicryl 3-0) with or without drain. The skin is closed using surgical staples or running resorbable intracutaneous suture (Monocryl 4-0). 


\subsection{Indication of the Approach}

The IH approach provides access to aneurysms of DACA (usually for segment A2, A3), as those originating at the origin of callosomarginal artery from the pericallosal artery. This approach is also adequate for highly positioned ACoA aneurysms with superoposterior projection of the dome. The unilateral anterior IH approach has been proposed to clip unruptured small and medium sized ACoA aneurysms, as it requires minimal brain retraction and carries less risk on olfactory nerves.

\subsection{Limitation of the Approach}

The unilateral anterior IH approach has some limitations. Compared to the more frequently used pterional approach, it provides less clear proximal control on A1 portions of ACAs, especially when the ACoA aneurysm is large or it is projecting anteriorly. This is the reason why it has been proposed for unruptured small- and medium-sized ACoA aneurysms.

\subsection{Complication and How to Avoid}

When placing the burr holes along the median line, or during the craniotomy between the two burr holes, it is possible to injure superior sagittal sinus and cause copious venous bleeding. But such complication is very rare. In this case, bipolar coagulation is useless and sometimes harmful. The best way to stop the bleeding is the addition of hemostatic material such as Spongostan ${ }^{\circledR}$ or Surgice ${ }^{\circledR}$ overlaid by a cottonoid and mechanical compression over it.

The damage of bridging veins is another potential complication of IH approach. It can be minimized by selecting the wider space between two bridging veins as the entry point to the interhemispheric fissure. If a bridging vein is fissured, placing small pieces of Surgicel ${ }^{\circledR}$ and fibrin glue can stop the bleeding. Coagulation of the vein is the last choice in case of failure of conservative attempts.

Brain contusions due to retractors must be taken into account and can be avoided applying only gentle retraction. Appropriate subarachnoid dissection and CSF release usually give significant help.

CSF leakage represents a minor complication related to this approach and can be minimized by careful waterproof dural closure. The risk of epidural hematoma is also low and can be further reduced placing multiple tack-up sutures of dura and craniotomy edges. A central suture can be also placed between the dura and two small holes at the center of the bone flap.

\section{Bibliography}

1. El-Noamany H, Nakagawa F, Hongo K, Kakizawa Y, Kobayashi S. Low anterior interhemispheric approach-a narrow corridor to aneurysms of the anterior communicating artery. Acta Neurochir (Wien). 2001;143(9):885-91.

2. Hayashi N, Sato H, Akioka N, Kurosaki K, Hori S, Endo S. Unilateral anterior interhemispheric approach for anterior communicating artery aneurysms with horizontal head position - technical note. Neurol Med Chir (Tokyo). 2011;51(2):160-3.

3. Hernesniemi J, Dashti R, Lehecka M, Niemela M, Rinne J, Lehto $\mathrm{H}$, et al. Microneurosurgical management of anterior communicating artery aneurysms. Surg Neurol. 2008;70(3):8-29.

4. Hori T, Kawamata T, Amano K, Aihara Y, Ono M, Miki N. Anterior interhemispheric approach for 100 tumors in and around the anterior third ventricle. Neurosurgery. 2010;66(ONS Suppl 1):ons65-74.

5. Lehecka M, Dashti R, Hernesniemi J, Niemela M, Koivisto T, Ronkainen A, et al. Microneurosurgical management of aneurysms at the A2 segment of anterior cerebral artery (proximal pericallosal artery) and its frontobasal branches. Surg Neurol. 2008;70(3):232-46.

6. Levy ML, Berto S. Interhemispheric transcallosal transchoroidal approach to the third ventricle. In: Nader R, Gragnaniello C, Berta SC, Sabbagh AJ, Levy ML, editors. Neurosurgery tricks of the trade. 1st ed. New York: Thieme; 2013. p. 19.

7. Oda J, Kato Y, Chen SF, Sodhiya P, Watabe T, Imizu S, et al. Intraoperative near-infrared indocyanine greenvideoangiography (ICG-VA) and graphic analysis of fluorescence intensity in cerebral aneurysm surgery. J Clin Neurosci. 2011;18:1097-100. 
Open Access This chapter is licensed under the terms of the Creative Commons Attribution 4.0 International License (http://creativecommons.org/licenses/by/4.0/), which permits use, sharing, adaptation, distribution and reproduction in any medium or format, as long as you give appropriate credit to the original author(s) and the source, provide a link to the Creative Commons license and indicate if changes were made.

The images or other third party material in this chapter are included in the chapter's Creative Commons license, unless indicated otherwise in a credit line to the material. If material is not included in the chapter's Creative Commons license and your intended use is not permitted by statutory regulation or exceeds the permitted use, you will need to obtain permission directly from the copyright holder.

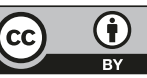

